\title{
Chattering-free Sliding Mode Control with Unmodeled \\ Dynamics
}

\author{
Don Krupp \\ Guidance and Control Division \\ Marshall Space Flight Center \\ MSFC, Alabama 35812 \\ 256-544-1812 don.krupp@msfc.nasa.gov \\ and \\ Yuri B. Shtessel \\ Department of Electrical and Computer Engineering \\ University of Alabama in Huntsville \\ Huntsville, Alabama 35899 \\ 256-890-6164 shtessel@ebs330.eb.uah.edu
}

\begin{abstract}
Sliding mode control systems are valued for their robust accommodation of uncertainties and their ability to reject disturbances. In this paper, a design methodology is proposed to eliminate the chattering phenomenon affecting sliding mode controlled plants with input unmodeled actuator dynamics of second order or greater. The proposed controller design is based on the relative degrees of the plant and the unmodeled actuator dynamics and the ranges of the uncertainties of the plant and actuator. The controller utilizes the pass filter characteristics of the physical actuating device to provide a smoothing effect on the discontinuous control signal rather than introducing any artificial dynamics into the controller design thus eliminating chattering in the system's output response.
\end{abstract}




\title{
Chattering-free Sliding Mode Control with Unmodeled Dynamics
}

\author{
Don Krupp \\ Guidance and Control Division \\ Marshall Space Flight Center \\ MSFC, Alabama 35812 \\ and \\ Yuri B. Shtessel \\ Department of Electrical and Computer Engineering \\ University of Alabama in Huntsville \\ Huntsville, Alabama 35899
}

\begin{abstract}
Sliding mode control systems are valued for their robust accommodation of uncertainties and their ability to reject disturbances. In this paper, a design methodology is proposed to eliminate the chattering phenomenon affecting sliding mode controlled plants with input unmodeled actuator dynamics of second order or greater. The proposed controller concept is based on the relative degrees of the plant and the unmodeled actuator dynamics and the ranges of the uncertainties of the plant and actuator. The controller utilizes the pass filter characteristics of the physical actuating device to provide a smoothing effect on the discontinuous control signal rather than introducing any artificial dynamics into the controller design thus eliminating chattering in the system's output response.
\end{abstract}

\subsection{Introduction}

Sliding mode control (SMC) systems are valued for their robust accommodation of uncertainties and their ability to reject disturbances. These qualities are especially important for flight control of space vehicles and aircraft where the control system must accommodate plant uncertainties and variations and reject disturbances from the external environment. Another attractive feature of SMC is the attainment of robust output tracking accomplished using a simple discontinuous switching element. One area of concern in implementing a sliding mode controller for flight or robot control is the reaction of the SMC to unmodeled dynamics and the elimination of chattering.

In the ideal case, the control signal generated by the SMC is discontinuous and switches at infinity frequency with finite amplitude. Since the actual implementation of an ideal switching element is impossible, various approximations to the ideal switch may lead to severe degradation of the system's performance. Additionally, the intentional introduction of a high-gain continuous approximation to the ideal switching element may actually lead to stable or unstable lower frequency limit cycle behavior. The chattering phenomenon is described as less than infinity frequency oscillations in the system's output response.

Many authors have proposed solutions to the chattering phenomenon. Utkin proposes to introduce an observer and a control such that sliding motion occurs on a manifold in the observer states. The discontinuous control is filtered by the unmodeled dynamics in the system and the result is a close approximation to the equivalent control [1]. Bartolini proposes to introduce an integrator into the controller and design a discontinuous control as the derivative of the actual control signal. The discontinuous control is passed through the artificially introduced integrator and the control signal passed to the plant is then continuous in nature [2], [3]. Fridman addresses the chattering problem by analyzing the saturation function as an approximation to the discontinuous switching element in the presence of singularly perturbed actuator dynamics [4]. Among the alternatives to eliminate chattering, the most promising are those which produce a continuous signal to the system. Other authors have shown that this approach produces satisfactory results if the unmodeled dynamics are of first order [5], [6]. If the unmodeled dynamics are of higher than first order, then the system response will exhibit limit cycle behavior and the task becomes one of analysis to assess the impact.

For many classes of systems, the control signal is acted upon by physical devices, which are stable and can be described as pass filters. While often the dynamics associated with these devices are known, it would be advantageous to treat them as unmodeled dynamics when considering the control algorithm design. We propose in this paper a technique to utilize the pass filter characteristics of the physical devices to smooth the discontinuous control input to eliminate chattering output response while retaining all the advantages associated with discontinuous sliding mode controllers.

\subsection{Problem Formulation}

Assume the mathematical model of the system under consideration is given by: 


$$
\begin{aligned}
& \dot{x}=\left[f_{0}(x)+\Delta f(x)\right]+\left[g_{0}(x)+\Delta g(x)\right] v \\
& y=h(x)
\end{aligned}
$$

where, $x \in \mathfrak{R}^{\mathrm{a}}$ are the system states, $v \in \mathfrak{R}^{1}$ is the control actually applied to the system and $y \in \Re^{1}$ is the output of the system. The vector fields $f, g$, and $h$ in (1) are possibly nonlinear and $f_{0}(x)$ and $g_{0}(x)$ represent a nominal value and $\Delta f(x)$ and $\Delta g(x)$ represent a bounded, uncertain term. This system is assumed to have relative degree $r=n$ and have states, $x$, which are measurable. If the relative degree $r<n$, we assume that the internal dynamics of the plant (1) are stable.

The system also contains actuators between the commanded control $u$ and the control actually applied to the system $v$. While the characteristics of these actuators including the relative degree and the mathematical description are known, we would prefer not to include the actuator dynamics in the formulation of the controller and will consider the actuators to be unmodeled dynamics with regard to the controller design. The unmodeled, input actuator dynamics are described by:

$$
\begin{aligned}
& \dot{z}=\left[a_{0}(z)+\Delta a(z)\right]+\left[b_{0}(z)+\Delta b(z)\right] u \\
& v=c z
\end{aligned}
$$

where, $z \in \Re^{k}$ are the unmodeled actuator states, $u \in$ $\Re^{\prime}$ is the commanded control and where $a_{0}(z)$ and $b_{0}(z)$ represents a nominal value and $\Delta a(z)$ and $\Delta b(z)$ represents a bounded, uncertain term. Additionally, while the vector fields $a, b$ and $c$ are partially known and possibly nonlinear, the order of the system of equations and the ranges of actuator parameters describing the actuator are known. The dynamics of the actuator are assumed to be stable, not singularly perturbed and have relative degree $r=k$. We also assume that while the output of the actuator is measurable, the actuator states, $z$, are not measurable. The problem is then to design a sliding mode control $u$ to robustly track a real-time reference profile, $\quad \lim \rightarrow \infty y_{\text {rot }}-y \mid=0$, with unmodeled dynamics of second or greater order between the commanded control, $u$, and control actually applied, $v$. By designing control $u$ to be discontinuous, we retain all the advantages of sliding mode controllers including rejecting disturbances and robustness to uncertainties. The actuator dynamics will not be included in the controller design, but the pass filter characteristics of the actuators will be utilized to smooth the control command u providing chattering free motion.

\subsection{Problem Solution}

We now propose a methodology, which consists of designing two sliding manifolds, a control function to provide asymptotic stability of the system and a sliding mode observer to robustly provide estimates to the derivatives of a sliding manifold.

\subsection{System Transformation}

The system given in (1) can be transformed to regular form [7]:

$$
\begin{aligned}
& \dot{\xi}=\xi_{2} \\
& \dot{\xi}_{2}=\xi_{3} \\
& \vdots \\
& \dot{\xi_{1}}=\phi(x)+\gamma(x) v \\
& \xi_{1}=y
\end{aligned}
$$

where $\phi(x)=\mathrm{L}_{1}^{\mathrm{a}}(h)$ and $\chi(x)=\mathrm{L}_{\mathrm{g}}\left(\mathrm{L}_{f}^{(m-1)}(h)\right)$ are the Lie derivatives and

$$
\phi(x)=\phi(x)+\Delta \phi(x), \gamma(x)=\gamma_{0}(x)+\Delta \gamma(x)
$$

It is assumed that $\gamma(x) \neq 0$ in a reasonable domain $x \in G$.

\subsection{Sliding Surface Design}

The sliding surface is designed for (3) assuming that the system's relative degree $r=n$ and disregarding the unmodeled input actuator dynamics [1].

$$
\sigma=e^{(n-1)}+C_{n-2} e^{(n-2)}+\ldots+C_{1} e^{(1)}+C_{0} e
$$

where $e=y_{\text {rof }}-y$ and the coefficients $C_{\mathrm{n}-2}, \ldots, C_{1}, C_{0}$ are selected to provide the desired eigenvalue placement for the differential equation $\sigma=0$ to provide the desired motion of the system's trajectory to the origin. It is important to note that the order of the sliding surface and the selection of the coefficients do not depend on the unmodeled actuator dynamics. Our design methodology will account for these unmodeled dynamics later in the controller design. We wish to design a sliding mode controller to provide convergence to and motion in the sliding surface $\sigma=0$ because this represents the desired motion of the system's trajectory to the origin with invariance due to uncertainties and certain disturbances. Unfortunately, the control actually applied to the system $v$ is not available for the controller design. One obvious approach to this problem appears to be that the control $v$ could be designed as if it were indeed available. The control $v$ could then be applied to the system as the input to the actuators, $u$. While this may seem like a reasonable approach, the resulting system motion will actually exhibit limit cycle behavior with possibly catastrophic results. The actual solution is to design the control $u$ to provide the desired robustness and also to eliminate chattering at the system's output through the unmodeled actuator dynamics.

\subsection{System Motion in o-subspace}

Because the control actually applied to the system $v$ is not available for design, we need to describe the system's motion in the $\sigma$-subspace. We will design a sliding mode control function to provide asymptotic stability in $\sigma=0$ by differentiating the sliding surface $\sigma$ until the control $u$ appears taking into account the relative degree of the unmodeled actuator dynamics, $r=$ $\boldsymbol{k}$. 
The system's motion in the $\sigma$-subspace is described by

$$
\begin{aligned}
& \dot{\eta}=\eta \\
& \dot{\eta}_{z}=\eta \\
& \vdots \\
& \dot{\eta}_{k+1}=\Phi\left(x, z, y_{\omega}, \dot{y}_{\omega}, \ldots, y_{\sigma^{\prime}}^{(n+k)}, \xi, \xi, \ldots, \xi\right)+\Gamma(x, z) \\
& \eta=\sigma
\end{aligned}
$$

The functions $\Phi\left(x, z, y_{n v}, \dot{y}_{w}, \ldots, y_{v v}^{(n+k)}, \xi_{1}, \xi_{2}, \ldots, \xi_{w}\right)$ and $\Gamma(x, z)$ contain elements of uncertainties associated with both the plant and the actuator and can be modeled as having the form previously considered:

$$
\begin{gathered}
\Phi(x, z, \ldots)=\Phi_{0}(x, z, \ldots)+\Delta \Phi(x, z, \ldots), \\
\Gamma(x, z)=\Gamma_{0}(x, z)+\Delta \Gamma(x, z)
\end{gathered}
$$

where $\Phi_{0}(x, z, \ldots)$ and $\Gamma_{0}(x, z)$ are nominal terms and $\Delta \Phi(x, z, \ldots)$ and $\Delta \Gamma(x, z)$ are bounded, uncertain terms. In order to proceed with the design of control $u$, we will at least need to have knowledge of the ranges of the uncertainties for $\Delta \Phi(x, z, \ldots)$ and $\Delta \Gamma(x, z)$. Note that all uncertainties in both the system and actuator are contained in (5) and are therefore present in $\Phi(x, z, \ldots)$ and $\Gamma(x, z)$.

\subsection{Control Function}

We need to find a control function $u$ to asymptotically stabilize the output of (5) or in essence $\sigma=0$. We now choose to design a sliding mode controller since the system has significant uncertain and unmodeled terms. The SMC must account for the relative degree of the actuator dynamics in designing control $u$. An auxiliary sliding surface is designed for (5) since the control $u$ appears and has the form:

$$
S=\eta_{k+1}+K_{k-1} \eta_{k}+\cdot \cdot+K_{1} \eta_{2}+K_{0} \eta_{1}
$$

with the coefficients $K_{k-1}, \ldots, K_{1}, K_{0}$ selected to provide the desired eigenvalue placement for the differential equation $S=0$. The purpose of the auxiliary sliding surface is to asymptotically robustly drive the system's trajectory to the initial sliding surface $\sigma=0$ with a guaranteed quality. While we have previously assumed that the sliding surface $\sigma$ was measurable, we now assume for a while that the derivatives of the sliding surface $\eta_{2}, \ldots$, $\eta_{k+1}$ are measurable.

\section{A candidate Lyapunov function is introduced such that}

$$
V=0.5 s^{2}
$$

The control $u$ is designed [1] to provide convergence to and asymptotic stability of the auxiliary sliding manifold $S=0$ and consequently $\sigma=0$. The designed control will provide convergence to $S=0$ in a finite time and the system's motion will remain invariant to all uncertainties while on the auxiliary sliding manifold. The form of the control $u$ is given by:

$$
u=\hat{u}_{\infty q}+\rho \cdot \operatorname{sign}(S)
$$

where

$$
\hat{u}_{\infty}=\left[\frac{-1}{\Gamma_{0}(x, z)}\right]\left[K_{k-1} \eta_{k+1}+K_{k-2} \eta_{k}+\ldots+K_{0} \eta_{2}+\Phi_{0}(x, z)\right]
$$

$\rho>\frac{L+\beta}{1-\alpha}$

$\| \Delta \Phi \mid<L$ is the upper bound on the plant and actuator uncertainties,

$\alpha=\frac{\Delta \Gamma(x, z)}{\Gamma_{0}(x, z)},|\alpha|<1$ is the relative uncertainty and $\rho_{1}$ is

selected to provide the desired reaching time to $S=0$.

This control law guarantees finite reaching time to the auxiliary sliding surface $S=0$ and has all the benefits associated with discontinuous sliding motion such as robustness to uncertainties and matched disturbances. After the auxiliary surface $S=0$ is reached, the system's motion is asymptotically driven to the sliding surface $\sigma=0$. Similarly, after $\sigma=0$ is reached, the system's motion has guaranteed quality and is asymptotically driven to the origin. The significance of this methodology is that the system's motion follows the prescribed motion of $\sigma=0$ and is robust to plant and actuator uncertainties which were not considered in the design of the sliding surface $\sigma=0$. Additionally, by utilizing the pass filter characteristics of the unmodeled actuator to smooth the discontinuous control $u$, chattering is eliminated in the system's output response.

\subsection{Sliding Mode Observer}

Now, since the derivatives of the auxiliary sliding surface are not typically measurable, we design a sliding mode observer to robustly provide estimates of these derivatives compensating for the uncertainties in the plant and actuator. The sliding mode observer is designed [8] and is given by:

$$
\begin{aligned}
& \hat{\sigma}_{1}=-L_{1} \operatorname{sign}\left(\hat{\sigma}_{1}-\sigma\right) \\
& \dot{w}_{1}=\left(\frac{1}{\tau}\right)\left[-w_{1}-L_{1} \operatorname{sign}\left(\hat{\sigma}_{1}-\sigma\right)\right] \\
& \hat{\sigma}_{2}=-L_{2} \operatorname{sign}\left(\hat{\sigma}_{2}-w_{1}\right) \\
& \dot{w}_{2}=\left(\frac{1}{\tau}\right)\left[-w_{2}-L_{2} \operatorname{sign}\left(\hat{\sigma}_{2}-w_{1}\right)\right] \\
& \vdots \\
& \hat{\sigma}_{k+1}=-L_{k} \operatorname{sign}\left(\hat{\sigma}_{k+1}-w_{k}\right)
\end{aligned}
$$

\subsection{Example}

The proposed design technique was applied to a linearized model of the pitch plane dynamics of an aircraft at Mach 0.7 and at an altitude of 10000 feet, modeled previously in the literature [9]. For this example, the model is described by 


$$
\begin{aligned}
& \dot{\alpha}=\left(Z_{a}\right) \alpha+\left(Z_{q}\right) q+\left(Z_{\delta}\right) \delta+\left(Z_{a}\right) \alpha_{w} \\
& \dot{q}=\left(M_{a}\right) \alpha+\left(M_{q}\right) q+\left(M_{\delta}\right) \delta+\left(M_{a}\right) \alpha_{w} \\
& \ddot{\delta}=-2 \zeta a \dot{\delta}-\omega^{2} \delta+\omega^{2} u \\
& y=q
\end{aligned}
$$

where, $\alpha$ is the angle of attack, $q$ is the pitch rate, $\delta$ is the elevon deflection angle (the control actually applied to the plant), $\alpha_{w}$ is the angle of attack perturbation due to wind gusts during flight and $y_{\text {sot }}$ is the reference pitch rate profile to track.

The sliding surface $\sigma$ is designed for (9) based on the relative degree of the system $r=1$ and disregarding the unmodeled actuator dynamics.

$$
\sigma=e=y_{\text {rot }}-y \quad(10)
$$

Now, the equation describing this sliding surface is differentiated to account for the relative degree of the unmodeled actuator dynamics $(r=2)$ to obtain the system's motion in $\sigma$-subspace.

$$
\begin{aligned}
& \dot{\eta}_{1}=\eta_{2} \\
& \dot{\eta}_{2}=\eta_{3} \\
& \dot{\eta}_{3}=\ddot{y}_{\sigma}-C_{\alpha} \alpha-C_{q} q-C_{\delta} \delta-Z_{\delta_{0}} u \\
& \eta_{1}=\sigma
\end{aligned}
$$

where

$$
\begin{aligned}
& C_{\alpha}=M_{\infty} Z_{\infty}^{2}+M_{\infty} M_{\infty} Z_{\infty}+M_{\infty}^{2} Z_{\phi 0}+M_{\infty}^{2} M_{\infty} \\
& C_{\phi}=M_{\infty} Z_{\infty} Z_{\infty}+2 M_{\infty} M_{\infty 0} Z_{\infty}+M_{\infty 0}^{3} \\
& C_{\delta}=M_{\infty} Z_{\infty} Z_{\infty}+M_{\infty} M_{\phi} Z_{\infty}+M_{\infty} M_{\infty} Z_{\phi}+M_{\phi}^{2} \\
& M_{\infty}
\end{aligned}
$$

The auxiliary sliding surface $S$ can then be designed for (11) to provide the desired quality of the system's motion toward the sliding surface $\sigma=0$.

$$
S=\eta_{3}+40 \eta_{2}+400 \eta_{1} \quad \text { (12) }
$$

The actual control function $u$ is designed to provide finite reaching time to the auxiliary sliding surface $S=$ 0 .

$$
u=\hat{u}_{\infty}-0.37 \operatorname{sign}(S)
$$

with

$$
\hat{u}_{\infty q}=\frac{\dddot{y}_{q \sigma}-C_{\alpha} \alpha-C_{q} q-C_{\delta} \delta}{Z_{s 0}}
$$

We have assumed that the sliding surface $\sigma\left(\eta_{1}\right)$ is measurable but the derivatives of the sliding surface $\left(\eta_{2}, \eta_{3}\right)$ are not measurable. Therefore, we need to estimate the derivatives of the sliding surface $\sigma$ to generate our auxiliary sliding surface $S$. In order to simplify the implementation of the sliding mode observer, a continuous form of the observer was designed.

\subsection{Simulation}

The model of the aircraft and the controller were simulated considering the reference signal to be tracked consisted of a series of pulses which were passed through a second-order filter. Multiple simulations were nun allowing the time-invariant plant and actuator parameters to vary as much as vary $\pm 15 \%$ of their nominal values. Additionally, there was a disturbance applied to the system as a perturbation to the system's angle of attack. This perturbation was modeled as a bias and pulses and could physically be caused by steady state winds and gusts during flight.

Figure 1 shows the result of designing a sliding mode controller to provide stability to the sliding surface $\sigma=$ 0 without accounting for the unmodeled actuator dynamics. This controller was designed with respect to $\sigma=0$ not $S=0$ and assuming the control $v$ was available. Obviously, the system's output exhibits limit cycle behavior and clearly could not be considered a successful design.

The remaining figures show the results of the application of our design methodology. Figure 2 shows the commanded pitch rate and the actual pitch rate for the series of pulses. The commanded and applied control signals are shown in Figures 3 and 4. Figure 4 shows that the control signal actually applied to the plant model is continuous thereby eliminating chattering and undesired limit cycle activity.

The simulation also investigated the possibility of generating the auxiliary sliding surface, $S=0$, without estimating the second derivative, $\eta_{3}$, of the sliding manifold, $\sigma=0$. The elimination of the higher order derivative means that we give up performance in the $\eta$ dynamics, however, we retain stability in the $S$ subspace. If the relative degree of the actuator is one, then neither the first or second derivatives, $\eta_{2}$ and $\eta_{3}$, are needed for stability and can be eliminated from the auxiliary manifold. If the relative degree of the actuator is two, then the second derivative of the sliding surface, $\eta_{3}$, is not required for stability in the $\eta$-dynamics. This result is general and can be proved for stable unmodeled dynamics with relative degree of one, $r=1$.

\subsection{Conclusions}

In this paper, a design methodology has been presented to eliminate the chattering phenomenon affecting sliding mode controlled plants with unmodeled actuator dynamics of second order or greater. The methodology is based on utilizing the pass filter characteristics of the physical actuating device to provide the smoothing effect to the discontinuous control signal. The resulting design provides robust output tracking for uncertainties in the plant and actuator without the added complexity of including the mathematical model of the actuator in the controller algorithm. 


\subsection{References}

[1] V. Utkin, Sliding Modes in Control Optimization, Springer-Verlag, 1992.

[2] G. Bartolini, P. Pydynowski, "An Improved, Chattering Free, V.S.C. Scheme for Uncertain Dynamical Systems," IEEE Transactions on Automatic Control, Vol. 41, No. 8, August 1996.

[3] G. Bartolini, A. Ferrara, E. Usai, "Chattering Avoidance by Second-Order Sliding Mode Control," IEEE Transactions on Automatic Control, Vol. 43, No. 2, February 1998.

[4] Fridman, Loonid, "Chattering in High Gain Control Systems with Fast Actuators," Proceedings of the $36^{\text {th }}$ Conference on Decision and Control, San Diego, California, December 1997.

[5] Y. Shtessel, Y., Lee, "New Approach to Chattering Analysis in Systems with Sliding Modes," Proceedings of $35^{\text {th }}$ Conference on Decision and Control, Kobe, Japan, December 1996.

[6] Chung, Steven and Lin, Chun-Liang, "A General Class of Sliding Surface for Sliding Mode Control," IEEE Transactions on Automatic Control, Vol. 43, No. 1, January 1998.

[7] Isidori, Alberto, Nonlinear Control Systems, Springer-Verlag, 1995.

[8] A. Rundell, S. Drakunov, R. DeCarlo, "A Sliding Mode Observer and Controller for Stabilization of Rotational Motion of a Vertical Shaft Magnetic Bearing." IEEE Transactions on Control System Technology, Vol. 4, No. 5, September 1996.

[9] Pachter, M., Chandler, P.R., and Mears, M., "Reconfigurable Tracking Control with Saturation," Journal of Guidance, Control and Dynamics, Vol. 18, No. 5, Sept. -Oct. 1995.

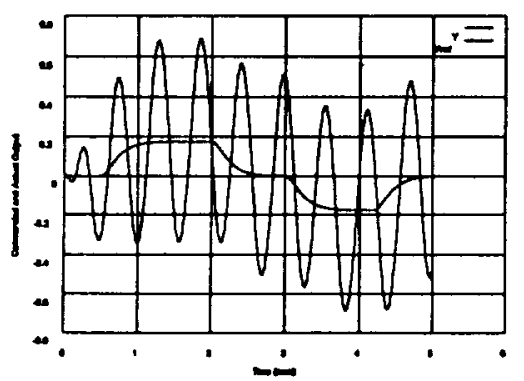

Figure 1: Controller Designed Without Accounting for Actuator Dynamics

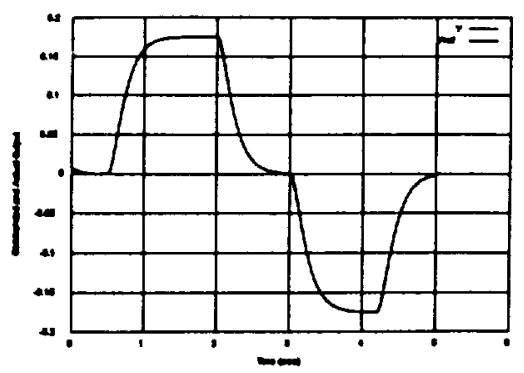

Figure 2: Controller Designed Accounting for Unmodeled Actuator Dynamics

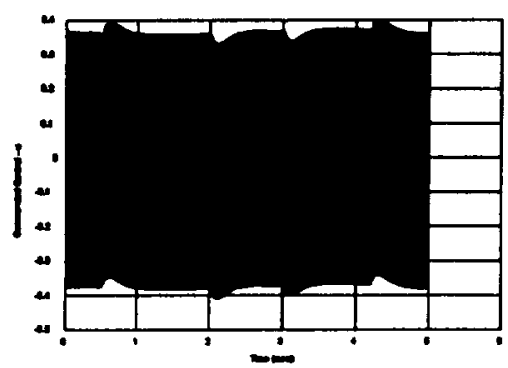

Figure 3: Commanded Control $u$

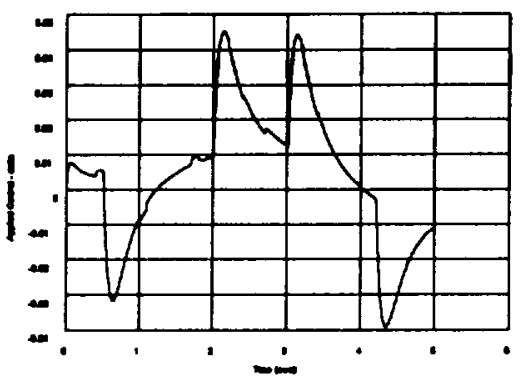

Figure 4: Control Actually Applied to Plant $v$ 